\title{
Ultrasonic guided wave detection of U-shaped pipeline
}

\author{
Longxiang Zhu ${ }^{1, *}$, Guangming Kong ${ }^{1}$, Haibing Zhang ${ }^{1}$, and Xiangyang $\mathrm{Yu}^{1}$ \\ ${ }^{1}$ Qingdao Campus of Naval Aviation University, Qingdao 266041, China
}

Keywords: Guided wave, U-shaped pipeline, Dispersion curve, Energy flow density, Detection sensitivity.

\begin{abstract}
The guided waves in the elbow was analyzed using semianalytical finite element method, which was used to calculate the dispersion curves, along with the vibration deformation and energy flow density distribution of the elbow cross-section. The $\mathrm{L}(0,1)$ mode guided wave was used to detect different defects in the U-shaped pipeline in experiment. The average experimental velocity of $\mathrm{L}(0,1)$ mode in $\mathrm{U}$-shaped pipeline was between the theoretical velocities of the straight pipe and the elbow, due to the different velocities in the two parts. The detection sensitivity difference of $\mathrm{L}(0,1)$ mode guided wave to defects in intrados and extrados of the elbow was analyzed, the experimental result showed that the reflected signal amplitude of defect in extrados was significantly larger than the defect in intrados, which was consistent with the energy flow density distribution in elbow cross-section.
\end{abstract}

\section{Introduction}

In the detection of pipelines using ultrasonic guided waves, the piping system often consists of straight pipes and curved pipes. As the propagation characteristics of the guided waves in curved pipes are different from those in straight pipes ${ }^{[1]}$, the propagation of guided waves in elbow-containing piping systems is more complicated than straight pipes. There exist phenomena such as transmission rate decline ${ }^{[2]}$, mode conversion ${ }^{[3-5]}$, elbow reflection ${ }^{[6]}$, etc. at the elbow, which cause difficulties in defects detection in elbow-containing piping systems. In addition, since the elbow loses the axial symmetry of the straight pipe, the reflection of defect in the elbow has different characteristics from the one in the straight pipe, which may cause interference in the defect detection.

The detection sensitivity difference of $\mathrm{T}(0,1)$ mode guided waves to defects at different positions of elbow was studied experimentally by $\mathrm{Q}^{[7]}$, the result showed that the crack in the middle of the elbow could inhibits mode conversion and shared the highest detection sensitivity. $\mathrm{ZHOU}^{[8]}$ studied the defect detection of elbow-containing pipeline using $\mathrm{L}(0,2)$ mode guided wave, it was found that it was easier to detect cracks in the extrados of the elbow while harder to detect those in the other locations. In this paper, the semi-analytical finite element method was used to analyze the guided waves in the elbow, and the $\mathrm{L}(0,1)$

\footnotetext{
*Corresponding author: jdyzzlx@,126.com
} 
mode guided wave was used to detect different defects in the U-shaped pipeline. The propagation characteristic of $\mathrm{L}(0,1)$ modal guided wave in the elbow was studied, and the detection sensitivity of $\mathrm{L}(0,1)$ mode guided wave to defects in different positions of the elbow was analyzed.

\section{Guide wave in the elbow}

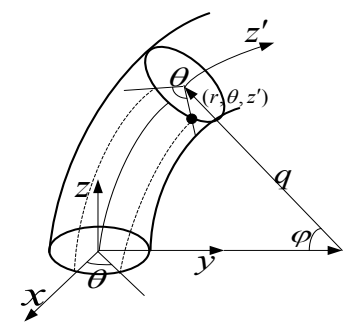

Fig. 1. Structure of elbow.

The study of guided waves in the elbow was based on the semi-analytical finite element method. When the guided wave propagates in the elbow, its propagation direction is along the axial direction (direction $z^{\prime}$ ), the governing equation can be derived from the virtual work principle ${ }^{[1]}$ :

$$
\int_{V} \delta \boldsymbol{\varepsilon}^{T} \boldsymbol{\sigma} d V+\int_{V} \delta \boldsymbol{u}^{T} \rho \ddot{\boldsymbol{u}} d V=0
$$

In equation (1), $\ddot{\boldsymbol{u}}$ is the second derivative of $\mathbf{u}$ versus time, $\rho$ is density. According to the propagation characteristic of guided wave, the displacement vector at an arbitrary point in the pipe can be written as:

$$
\boldsymbol{u}=\boldsymbol{U}(r, \theta) \exp \left(i k z^{\prime}\right) \exp (-i \omega t)
$$

In equation (2), $k$ is the wave number, $\omega$ is the angular frequency. Substituting equation (2) and equation (1), combined with elastic geometric equation and physical equation, we can get the guided wave dispersion equation in the elbow as follows:

$$
\left(\left[\begin{array}{cc}
0 & \mathbf{K}_{1}-\omega^{2} \mathbf{M} \\
\mathbf{K}_{1}-\omega^{2} \mathbf{M} & i\left(\mathbf{K}_{2}-\mathbf{K}_{2}^{T}\right.
\end{array}\right]-k\left[\begin{array}{cc}
\mathbf{K}_{1}-\omega^{2} \mathbf{M} & 0 \\
0 & -\mathbf{K}_{3}
\end{array}\right]\right)\left[\begin{array}{c}
\mathbf{U}_{1} \\
k \mathbf{U}_{1}
\end{array}\right]=\left[\begin{array}{l}
0 \\
0
\end{array}\right]
$$

where $\boldsymbol{K}_{1}, \boldsymbol{K}_{2}, \boldsymbol{K}_{3}, \boldsymbol{M}$ are matrices determined by elbow cross-section geometry, $\boldsymbol{U}_{1}$ is the nodal displacement vector for elbow cross-section. The equation is an eigenvalue equation, and each $k-\omega$ corresponds to each mode guided wave. The dispersion curves can be calculated by solving the equation. The nodal displacement vector for some $k-\omega$ is the displacement of each node on the elbow cross-section when some mode guided wave propagates. Using the nodal displacement vector, the vibration deformation and energy flow density distribution of the elbow cross-section can be calculated. 


\section{Experimental setup}

Figure 1 shows the U-shaped pipeline for experiment. The pipeline was composed of two straight pipes and a $180^{\circ}$ elbow. The bending radius of the elbow was $192.5 \mathrm{~mm}(\mathrm{q}=192.5 \mathrm{~mm})$, the length of the two straight pipes was $1290 \mathrm{~mm}$. The outer diameter of the pipeline section was $28 \mathrm{~mm}(d=28 \mathrm{~mm})$. The wall thickness was $3 \mathrm{~mm}(t=3 \mathrm{~mm})$. The material of the U-shaped pipeline was carbon steel, the density was $7932 \mathrm{~kg} / \mathrm{m}^{3}$, the elastic modulus was $217 \mathrm{GPa}$, and the Poisson's ratio was 0.3 .

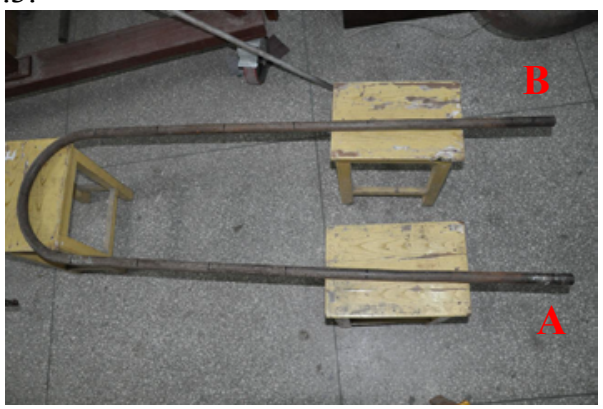

Fig. 1. U-shaped pipeline in experiment.

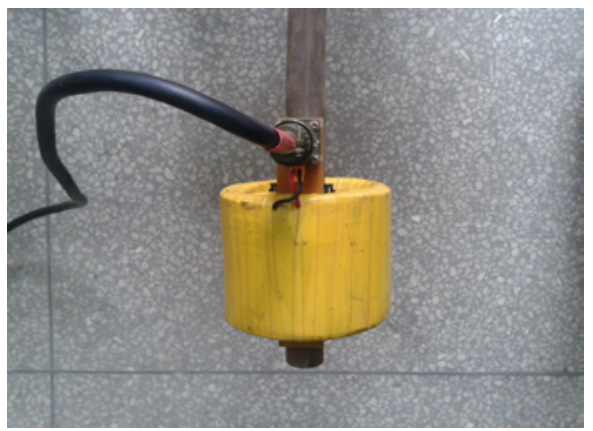

Fig. 2. magnetostrictive transducer

Figure2 shows the magnetostrictive sensor for longitudinal ultrasonic guided wave in experiment, placed at the A end of the U-shaped pipeline. The sensor was composed of a coil $^{[9]}$, when an alternating current signal was applied to the coil, the axially alternating stress was generated in the pipeline by the magnetostrictive effect of the ferromagnetic pipeline itself, and then longitudinal guided wave was generated in the straight pipe of the U-shaped pipeline. The guided wave propagated in the U-shaped pipeline and reflected in the end. When the longitudinal guided wave passed through the area where the sensor was located, the guided wave was converted into electrical signal for reception by the reverse magnetostrictive effect of the pipeline. The magnetizing coil was used to provide a bias magnetic field. The presence of biasing magnetic field could increase the sensitivity of the sensor and ensured a linear relationship between the electrical signal and the guided wave. 


\section{Detection for intact U-shaped pipeline}

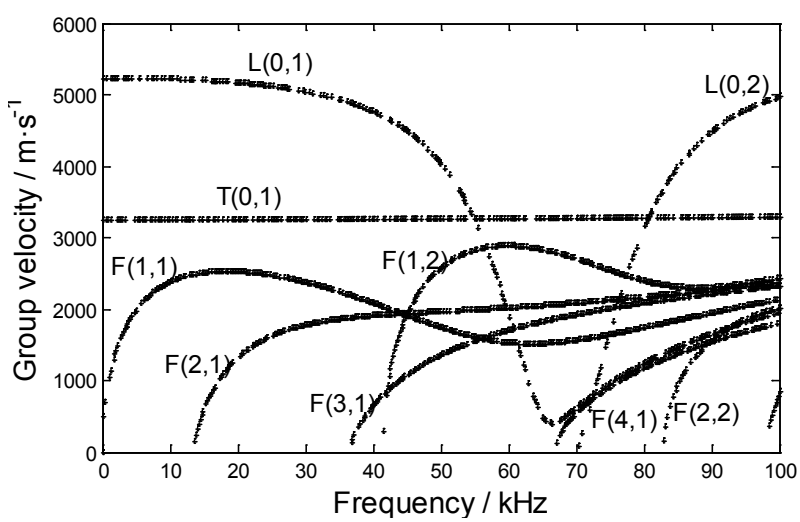

Fig. 3. Dispersion curves of straight part in U-shaped pipeline.

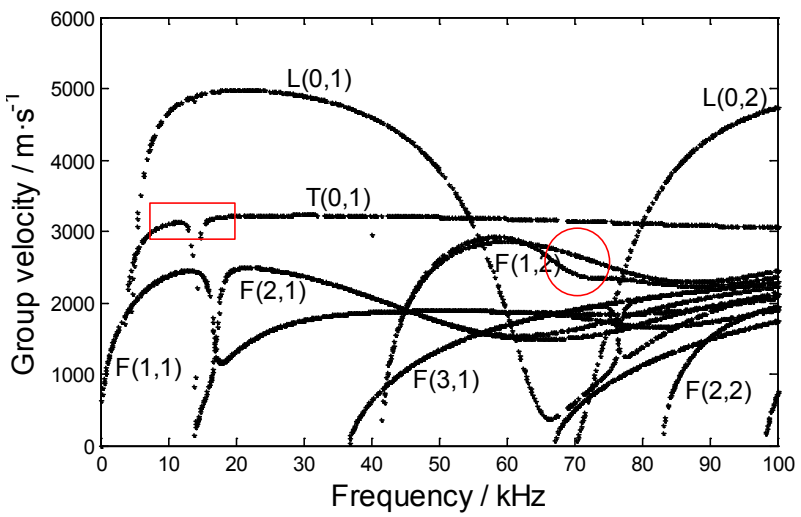

Fig. 4. Dispersion curves of elbow part in U-shaped pipeline.

Figure 3 and Figure 4 shows the guided wave dispersion curves in the straight pipe part and the elbow part of U-shaped pipeline respectively, calculated by the semi-analytical finite element method. Comparing the two, we can find that there were following characterictics for dispersion curves in the elbow. First, there existed cutoff frequencies for $\mathrm{L}(0,1)$ and $\mathrm{T}(0,1)$ modes in the elbow. Second, the group velocity of $\mathrm{T}(0,1)$ mode was no longer constant. Third, there was "modal branching" phenomenon for flexural mode ${ }^{[10]}$ (such as $F(1,2)$ ) in the elbow, as shown by the circular marks in Figure4. At last, There was "modal coupling" phenomenon in the elbow, as shown by the rectangular mark in Figure4, which also appeared in liquidfilled cylindrical shells ${ }^{[11]}$ and helical waveguide ${ }^{[12]}$.

Since the guided wave was generated by the sensor in the straight pipe section, according to the dispersion curve of the guided wave in the straight pipe, it could be known that in the low frequency region, the sensor generated the $\mathrm{L}(0,1)$ mode guided wave in the straight pipe. when the $\mathrm{L}(0,1)$ mode guided wave entered the elbow region, due to the difference of dispersion curves between the elbow and the straight pipe, the wave would propagate according to the characteristics of the $\mathrm{L}(0,1)$ mode in the elbow. When the wave passed through the elbow into the straight pipe, it would again propagate according to $L(0,1)$ mode in the straight pipe. Figure 5 is the cross-sectional deformation for the guided wave propagating in the elbow and in the straight pipe, the arrow in Fig. 5 points to the intrados of the elbow. It can be seen that the cross-section deformation of the $\mathrm{L}(0,1)$ mode in the straight 
pipe was axisymmetric, while the $\mathrm{L}(0,1)$ mode in the elbow lost axial symmetry, and its crosssectional deformation was similar to the $F(1,1)$ mode in the straight pipe.

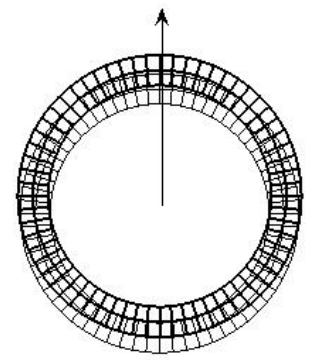

$\mathrm{L}(0,1)$ mode in the elbow

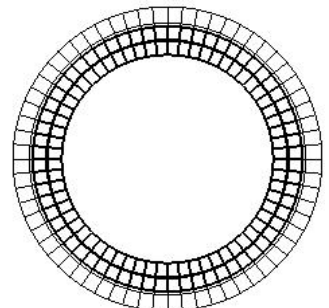

$\mathrm{L}(0,1)$ mode in straight pipe

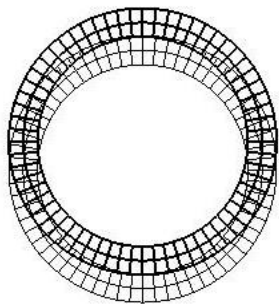

$\mathrm{F}(1,1)$ mode in straight pipe

Fig. 5. Cross-sectional deformation $(\mathrm{f}=10 \mathrm{kHz})$.

Figure6 shows the experimental signals for intact U-shaped pipeline at $30 \mathrm{kHz}$. According to the time interval of the end reflection signals, the average velocity of $\mathrm{L}(0,1)$ mode guided wave propagating in the U-shaped pipeline can be calculated, which was $4910 \mathrm{~m} / \mathrm{s}$ in experiment. According to the previous calculation, the theoretical velocity of $\mathrm{L}(0,1)$ mode in the straight pipe is $5030 \mathrm{~m} / \mathrm{s}$, and the theoretical velocity in the elbow is $4900 \mathrm{~m} / \mathrm{s}$, so the average experimental velocity was between the theoretical velocities of the straight pipe and the elbow. As defects were located by calculating the time difference of defect reflection signals traditionally in detection, this may cause positioning error due to the velocities difference.

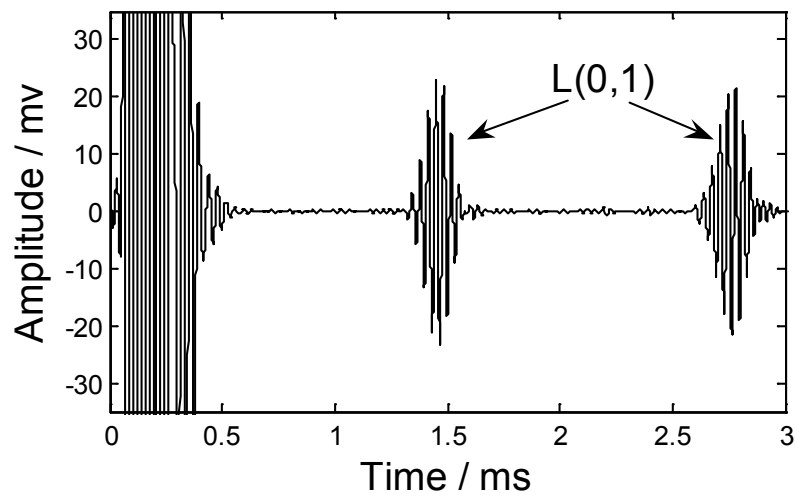

Fig. 6. Detecting signal for intact U-shaped pipeline $(\mathrm{f}=30 \mathrm{kHz})$.

\section{Detection for U-shaped pipeline with defect}

Two identical U-shaped pipelines were selected for defect detection experiment (hereinafter referred to as pipeline 1 and pipeline 2), and a notch defect was processed at the center of the elbow of the two pipelines. The dimensions of the defects were the same (length is $18 \mathrm{~mm}$, width is $2 \mathrm{~mm}$ in axial direction), but the circumferential positions were different, in which the defect of the pipeline 1 was located at the intrados of the elbow, the defect of the pipeline 2 was located at the extrados of the elbow, the two defects were all symmetric about the center line of intrados and extrados. 


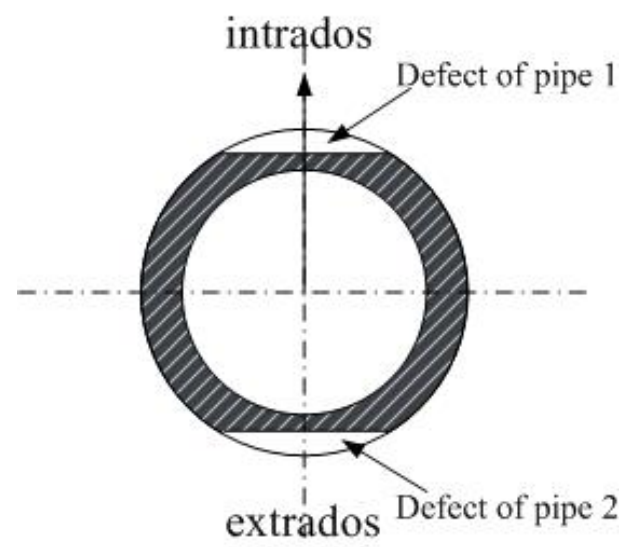

Fig. 7. Cross section of defect.

Fig. 8 and Fig. 9 show the detection signals obtained at $31 \mathrm{kHz}$ and $36 \mathrm{kHz}$. For defects of the same size, the amplitudes of the reflected signals were different for defects in different circumferential positions of the elbow. The reflected signal amplitude of defect in extrados was significantly larger than the defect in intrados, which was consistent with the energy flow density distribution in Figure 10 (the arrow in Fig.10 points to the intrados of elbow, so the energy density of the $\mathrm{L}(0,1)$ mode was higher in the extrados than in the intrados at $31 \mathrm{kHz}$ and $36 \mathrm{kHz}$ ). It could be concluded that since the energy flow density of $\mathrm{L}(0,1)$ mode no longer had axis symmetry, the unevenly distribution in intrados and extrados resulted in different detection sensitivities for defects in different circumferential positions.

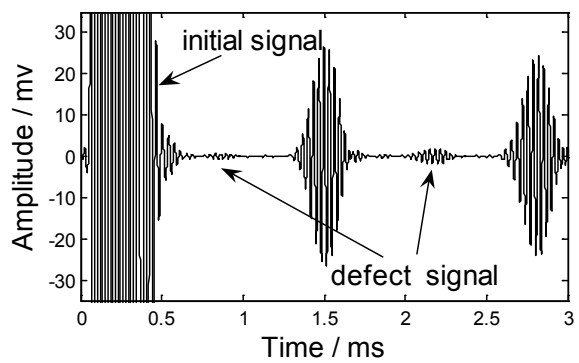

(a) Defect in intrados

Fig. 8. Signals detected in experiment $(\mathrm{f}=31 \mathrm{kHz})$

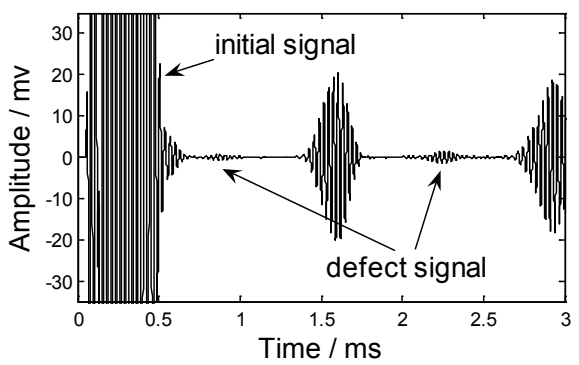

(a) Defect in intrados

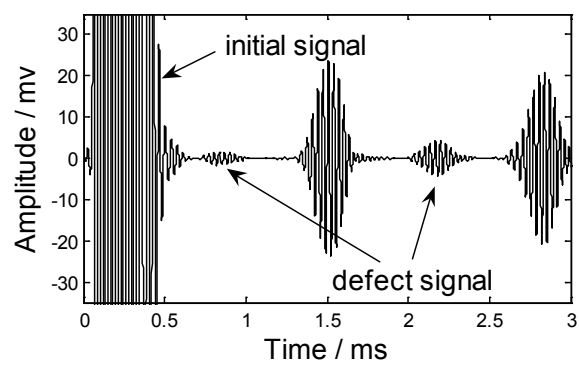

(b) Defect in extrados

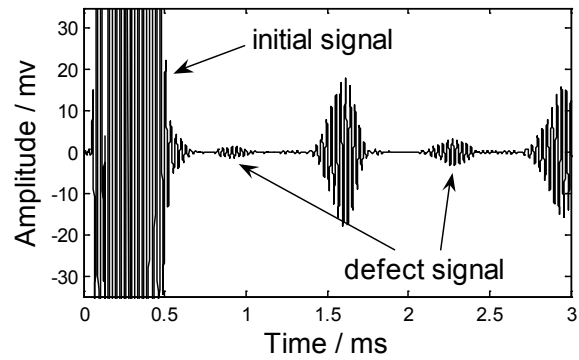

(b) Defect in extrados

Fig. 9. Signals detected in experiment $(\mathrm{f}=36 \mathrm{kHz})$. 


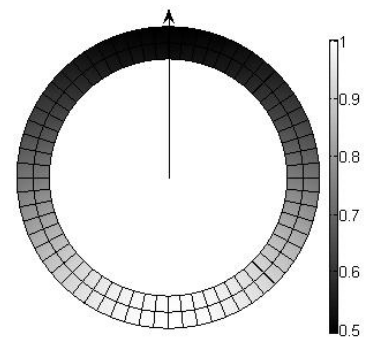

(a) $\mathrm{L}(0,1), \mathrm{f}=31 \mathrm{kHz}$

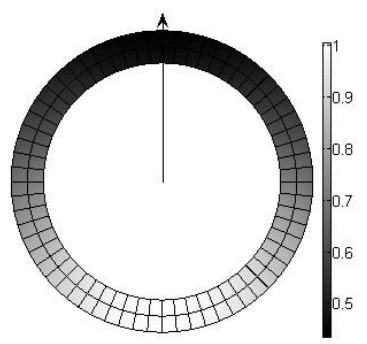

(b) $\mathrm{L}(0,1), \mathrm{f}=36 \mathrm{kHz}$

Fig. 10. Energy flow density distribution.

\section{Conclusion}

The L $(0,1)$ mode guided wave was analyzed using semi-analytical finite element method, and was used to detect $U$-shaped pipeline in experiment. As the $\mathrm{L}(0,1)$ mode in the elbow lost axial symmetry, there were differencies between the dispersion curves in elbow and straight pipe, which caused different velocities in the two parts. So the average experimental velocity in U-shaped pipeline was between the theoretical velocities of straight pipe and elbow. The defects in intrados and extrados of the elbow was detected, it was found that the reflected signal amplitude of defect in extrados was significantly larger than the defect in intrados, this means $\mathrm{L}(0,1)$ mode guided wave has different detection sensitivity to defects in intrados and extrados of the elbow. This phenomenon was consistent with the energy flow density distribution in elbow cross-section, while the energy density of the $\mathrm{L}(0,1)$ mode was higher in the extrados than in the intrados.

\section{References}

1. T. Hayashi, K. Kawashima, Z.Q. Sun, et al. Guided wave propagation mechanics across a pipe elbow[J]. Journal of Pressure Vessel Technology, 2005, 127: 322-327.

2. Y. Li, Y. Zou, S.N. Zhang, et al. Numerical and experimental research on $\mathrm{T}(0,1)$ guided waves propagating in bended pipes[J], Technical Acoustics, 2017, 36(5): 442-449

3. J. Park, J. Lee, S.G. Jeong, et al. A study on guided wave propagation in a long distance curved pipe[J]. Journal of Mechanical Science and Technology, 2019, 33(9): 1-7.

4. F. Simonetti, M.Y. Alqaradawi. Guided ultrasonic wave tomography of a pipe bend exposed to environmental conditions: A long-term monitoring experiment[J]. NDT and E International, 2019, 105: 1-10.

5. B. Verma, T.K. Mishra, K. Balasubramaniam, et al. Interaction of low-frequency axisymmetric ultrasonic guided waves with bends in pipes of arbitrary bend angle and general bend radius[J]. Ultrasonics, 2014, 54:801-808.

6. M. Bakkali, A. Lhemery, V Baronian, et al. A modal formulation for the propagation of guided waves in straight and curved pipes and the scattering at their junction[J]. Journal of Physics: Conference Series, 2014, 498:1-11.

7. M.X. Qi, S.P. Zhou, J. Ni, et al. Investigation on ultrasonic guided waves propagation in elbow pipe[J]. International Journal of Pressure Vessels and Piping, 2016, 1-6.

8. S.P Zhou, P.G. Zhang, W.C. Lv, et al. Detection of cracks in elbow pipes using guided waves[J]. Journal of Mechanical Engineering, 2015, 51(6): 58-65 
9. H. Kwun, "Method and apparatus generation and detecting torsional wave inspection of pipes or tubes". U. S. Patent 6429650 B1, 2002.

10. A. Demma, P. Cawley, M.J.S. Lowe, et al. The effect of bends on the propagation of guided waves in pipes[J]. Journal of Pressure Vessel Technology, 2005, 127: 328-335.

11. H. Kwun, K.A. Bartels, C. Dynes. Dispersion of longitudinal waves propagating in liquid-filled cylindrical shells[J]. J.Acoust.Soc.Am., 1999, 105(5): 2601-2611.

12. F. Treyssede. Elastic waves in helical waveguides[J]. Wave Motion, 2008, 45: 457-470. 